\title{
Neutron stars with submillisecond periods: a population of high mass objects?
}

\author{
Luciano Burderi ${ }^{1}$, Andrea Possenti ${ }^{2}$, Monica Colpi ${ }^{3}$, Tiziana Di Salvo ${ }^{1}$, Nichi D'Amico ${ }^{4}$
}

To appear in The Astrophysical Journal, June 1999

\begin{abstract}
Fast spinning neutron stars, recycled in low mass binaries, may have accreted a substantial amount of mass. The available relativistic measurements of neutron star masses, all clustering around $1.4 M_{\odot}$, however refer mostly to slowly rotating neutron stars which accreted a tiny amount of mass during evolution in a massive binary system.

We develop a semi-analytical model for studying the evolution of the spin period $P$ of a magnetic neutron star as a function of the baryonic mass load $M_{a c}$; evolution is followed down to submillisecond periods and the magnetic field is allowed to decay significantly before the end of recycling. We use different equations of state and include rotational deformation effects, the presence of a strong gravitational field and of a magnetosphere. For the non-magnetic case, comparison with numerical relativistic codes shows the accuracy of our description.

The minimum accreted mass requested to spin-up a magnetized $1.35 M_{\odot}$-neutron star at a few millisecond is $\sim 0.05 M_{\odot}$, while this value doubles for an unmagnetized neutron star. Below 1 millisecond the request is of at least $\sim 0.25 M_{\odot}$. Only highly non-conservative scenarios for the binary evolution could prevent the transfer of such a mass to the compact object. Unless a physical mechanism limits the rotational period, there may exist a yet undetected population of massive submillisecond neutron stars. The discovery of a submillisecond neutron star would imply a lower limit for its mass of about $1.7 M_{\odot}$.
\end{abstract}

Subject headings: accretion, accretion disks — equation of state — pulsars: general — relativity - stars: neutron

\section{Introduction}

The evolution of a neutron star (NS) in a binary system differs considerably in the case the donor star is a high mass giant star in a HMXB or is a lighter star in a LMXB (see e.g. Verbunt 1993; Lipunov 1992). It is only in the former case that the formation of a pulsar (slowly rotating at $P>50 \mathrm{~ms}$ ) in a relativistic NS-NS binary allows for the accurate estimate of the masses of the two components (Portegies, Zwart \& Yungelson 1998). Collecting all data for the known binary pulsars, Thorsett \& Chakrabarty (1998) found

\footnotetext{
${ }^{1}$ Dipartimento di Scienze Fisiche ed Astronomiche dell'Universita', via Archirafi 36, 90123 Palermo, Italy

${ }^{2}$ Dipartimento di Astronomia dell'Universita', via Zamboni 33, 40126 Bologna, Italy

${ }^{3}$ Dipartimento di Fisica dell'Universita', via Celoria 16, 20133 Milano, Italy

${ }^{4}$ Osservatorio Astronomico di Bologna, via Zamboni 33, 40126 Bologna, Italy
} 
an average mass of $1.35 M_{\odot}$ (with a very narrow spread $\sigma=0.04 M_{\odot}$ ) for both NSs in these systems. These values are fully compatible with the two hypotheses: that NSs at birth possess a canonical mass of about $1.4 M_{\odot}$ (Woosley \& Weaver 1986; but see Timmes, Woosley \& Weaver 1996) and that the HMXB phase is shortlived so that at most $10^{-2} M_{\odot}$ can be accreted onto the oldest nonpulsating NS (Bhattacharya \& van den Heuvel 1991). On the other hand, it is widely accepted (Phinney \& Kulkarni 1994, van den Heuvel \& Rappaport 1992) that the formation of millisecond pulsars (MSPs; with period $P<10 \mathrm{~ms}$ ) takes place inside LMXBs, where an old neutron star is spun-up by accretion torques. The mass load can be significant, in these systems, and, in principle, the NS can reach ultrashort periods $P \lesssim 1$ ms (depending on the equation of state) on a time that is controlled by the evolution of the magnetic field (Possenti et al. 1998). Thus, there is a close relationship between the spin period $P$, the gravitational mass and the magnetic field evolution.

Cook, Shapiro \& Teukolsky (1994a: CSTa) developed a relativistic code to study the spin evolution of an unmagnetized NS accreting from the inner edge of a Keplerian disk. Here we reproduce their results using a semi-analytical model (described in section $\S 2$ ). Then we allow for the presence of a magnetosphere around the NS and explore the consequences on the relation between the baryonic mass load $M_{a c}$ and the spin rate $P$, considering two scenarios for the magnetic field evolution. The minimum mass load requested to reach the submillisecond range is computed for a magnetic neutron star in section $\S 3$, adopting different equations of state (EoSs). Observational tests are discussed in section $\S 4$; in $\S 5$ we present our conclusions.

\section{Mass loading and spin evolution}

In this section we shortly describe the semi-analytical model used to compute the $P$ vs $M_{a c}$ relation, which is estimated down to limiting periods $\lesssim 1$ millisecond. We first derive a simple equation describing the evolution of the gravitational mass $M_{G}$ as a function of the baryon load. We then introduce the set of equations for the evolution of the rotational frequency $\Omega$, including rotational effects on the NS radius during recycling. The angular momentum transfer rate is computed including the corrections due to a strong gravitational field. The equations are solved numerically.

The code follows the evolution of an NS of initial gravitational mass $M_{G, 0}=1.4 M_{\odot}$ which is accreting at a constant rate $\dot{m}_{B}$. The initial values of the NS baryonic mass $M_{B, 0}$ and of its radius $R_{0}$ are known once we specify the EoS (see e.g. Cook, Shapiro \& Teukolsky 1994b; CSTb). In our model, the gravitational and the baryonic masses $M_{G}$ and $M_{B}$ of the NS are related, at any given time, by $M_{G}=M_{B}(1-\alpha / R)$, where $R$ is the NS radius circumferential radius (i.e. the proper circumferential length at the NS equator divided by $2 \pi)$ and $\alpha$ is a constant evaluated using the initial set of parameters. Accordingly,

$$
M_{G}=M_{B}\left[1+\left(\frac{M_{G, 0}}{M_{B, 0}}-1\right)\left(\frac{R_{0}}{R}\right)\right]
$$

The decrease in radius due to the mass load is described using the simple relation $R \propto M_{G}^{-1 / 3}$ (for equilibria of degenerate nonrelativistic neutrons): with this scaling we estimate the non-rotating NS circumferential radius $R$ at any stage of the accretion. The evolution equation for $M_{G}$ thus reads:

$$
\dot{M}_{G}=\psi \dot{m}_{B}=\left[1+\left(\frac{M_{G, 0}}{M_{B, 0}}-1\right)\left(\frac{M_{G}}{M_{G, 0}}\right)^{1 / 3}\right]\left[1-\frac{M_{B}}{3 M_{G}}\left(\frac{M_{G, 0}}{M_{B, 0}}-1\right)\left(\frac{M_{G}}{M_{G, 0}}\right)^{1 / 3}\right]^{-1} \dot{m}_{B} .
$$

The mass increase is accompanied by the increase in the rotational frequency $\Omega$, and at any time (i.e., at any current value of $M_{G}$ ) we need to determine whether $\Omega$ is below or above the mass shedding limit 
$\Omega_{\max }$ that is the limiting angular frequency at which the gravitational pull is balanced by the centrifugal forces. $\Omega_{\max }$ is estimated using the classical expression

$$
\Omega_{\text {max }}=\Omega_{K}=\left(\frac{G M_{G}}{R_{\Omega, \text { max }}^{3}}\right)^{1 / 2}
$$

where $R_{\Omega, \max }$ denotes the circumferential radius of the rotating NS which in turn depends upon $\Omega_{\max }$. $R_{\Omega, \max }$ is estimated fitting the results of CSTb:

$$
R_{\Omega, \max }=1.5 R_{0}
$$

where $R_{0}$ is the radius of the static configuration at the current value $M_{G}$. In table 1 we compare equation (3) with the results of CSTb, derived using a relativistic code for 13 different EoSs $\left(M_{G}=1.4 M_{\odot}\right)$. The agreement is $\leq 5 \%$. The maximum angular speed occurs when the NS equatorial speed reaches the classical Keplerian limit computed taking $M_{G}$ as gravitational mass and as radius $R_{\Omega, \text { max }}$ : the rapid rotation inflates and deforms the NS surface. While the non-spherically-symmetric deformations (oblateness effects) are important in determining the radius of the spinning NS, they are almost irrelevant in changing the gravitational field felt by a test particle at the NS equator with respect to the corresponding spherical configuration. This is probably due to the fact that most of the mass is concentrated well inside the external radius, where the deformation effects due to rotation are almost negligible. This is predicted by the so called Roche Model (see e.g. Shapiro \& Teukolsky 1983), that, in the hypothesis outlined above, conclude that the maximum expansion of a uniformly rotating star along its equator is a factor of $3 / 2$, irrespective of the EoS adopted.

If $\Omega<\Omega_{\text {max }}$, spin up can proceed and the circumferential radius $R_{\Omega}$ of the rotating NS further increases. $R_{\Omega}$ is computed using the relation

$$
\log _{10}\left(R_{\Omega} / R_{0}\right)=\frac{1-\left(1-\Omega / \Omega_{\max }\right)^{1 / 3}}{6.75}
$$

derived fitting the results of CSTb. In Fig. 1 we report the values of $R_{\Omega}$ vs $\Omega$ from CSTb, for 5 EoSs $\left(M_{B}=1.4 M_{\odot}\right)$ and the relation given by equation (5). In the numerical scheme, $\Omega_{\max }$ is firstly determined from (3) using equation (4) for $R_{\Omega, \max }$. Then (5) is used to calculate $R_{\Omega}$.

\subsection{Unmagnetized case}

During the accretion process the specific angular momentum $l_{\text {in }}$ of the accreting matter is entirely transferred to the NS. From the angular momentum conservation $d\left(I_{\Omega} \Omega\right) / d t=\dot{m}_{B} l_{\text {in }}$ we obtain

$$
\dot{\Omega}=\frac{\dot{m}_{B}}{I_{\Omega}} \quad\left(l_{i n}-\psi \Omega \frac{\partial I_{\Omega}}{\partial M_{G}}\right)\left(1+\frac{\Omega}{I_{\Omega}} \frac{\partial I_{\Omega}}{\partial R_{\Omega}} \frac{\partial R_{\Omega}}{\partial \Omega}\right)^{-1}
$$

where $I_{\Omega}$ is the moment of inertia of the neutron star. Taking into account the inflation of the radius caused by the rapid rotation, we extrapolate a result of Ravenhall \& Pethick (1994) obtained for static stars:

$$
I_{\Omega}=0.21 M_{G} R_{\Omega}^{2}\left(1-\frac{2 G M_{G}}{R_{\Omega} c^{2}}\right)^{-1}
$$

The value $I_{\Omega}$ is significantly altered at high rotational rates. Hence, its derivatives with respect to $\Omega$ and $M_{G}$ cannot be neglected in the equation for $\dot{\Omega}$. 
The inner rim of the disk at $r_{i n}$ is at few NS gravitational radii, where relativistic gravity is important. Therefore the specific angular momentum $l_{\text {in }}$ carried by matter differs from the classical newtonian value $\sqrt{G M_{G} r_{i n}}$. Assuming an accretion disk co-rotating with the NS, and the Kerr metric for the approximate description of gravity around a NS (see Shibata \& Sasaki 1998 for a more detailed analysis of the metric), we obtain from Bardeen (1972):

$$
l_{i n}=c \frac{r_{h}^{1 / 2}\left(r_{i n}^{2}-2 a r_{h}^{1 / 2} r_{i n}^{1 / 2}+a^{2}\right)}{r_{i n}^{3 / 4}\left(r_{i n}^{3 / 2}-3 r_{h} r_{i n}^{1 / 2}+2 a r_{h}^{1 / 2}\right)^{1 / 2}}
$$

where $r_{h}=G M_{G} / c^{2}$ and $a=I_{\Omega} \Omega / M_{G} c$.

For an unmagnetized neutron star the inner rim $r_{i n}$ of the accretion disk either skims the NS surface or, for very compact NS, is truncated at the radius of the last stable orbit $r_{m s}$. Therefore the radius $r_{i n}$ is defined as:

$$
r_{i n}=\left\{\begin{array}{cl}
r_{m s} & \text { if } r_{m s}>R_{\Omega} \\
R_{\Omega} & \text { if } r_{m s} \leq R_{\Omega}
\end{array}\right.
$$

In the Kerr metric $r_{m s}$ is given by:

$$
r_{m s}=r_{h}\left(3+Z_{2}-\left[\left(3-Z_{1}\right)\left(3+Z_{1}+2 Z_{2}\right)\right]^{1 / 2}\right)
$$

with

$$
\begin{gathered}
Z_{1}=1+\left[1-\left(a / r_{h}\right)^{2}\right]^{1 / 3}\left[\left(1+a / r_{h}\right)^{1 / 3}+\left(1-a / r_{h}\right)^{1 / 3}\right] \\
Z_{2}=\left[3\left(a / r_{h}\right)^{2}+Z_{1}^{2}\right]^{1 / 2}
\end{gathered}
$$

Solving for the differential equations (2) and (6), we determine the NS rotational evolution under the hypothesis of stationary accretion. Our results are in agreement with the results of the relativistic code of CSTa, within $8 \%$ even in the high spin regime (see e.g. Fig. 2). The related computational effort is greatly reduced with respect to a fully relativistic numerical approach.

\subsection{Magnetized case}

For the case of a magnetized NS, there is another characteristic length, i.e., the magnetospheric radius $r_{m a g}$, which has to be compared with $r_{m s}$ and $R_{\Omega}$ when calculating the value of $r_{i n}$. The magnetospheric radius is computed as the product of the Alfven radius (see e.g. Lipunov 1992) times a factor $\phi \sim 1$, estimated as in Burderi et al. (1998). For high enough magnetic moment $\mu, r_{m a g}$ exceeds both $R_{\Omega}$ and $r_{m s}$ and the magnetic coupling between the disk and the star determines the extent of angular momentum transfer. The angular momentum balance relation in this case reads as $d\left(I_{\Omega} \Omega\right) / d t=g \dot{m}_{B} l_{i n}$, where the torque function $g=g(\Omega)$ accounts for the details of the interaction between the NS magnetosphere and the accretion disk. When $g=0$ the NS is on the so-called "spinup line", where it can load mass without modifying its angular momentum $I_{\Omega} \Omega$. To bracket the uncertainties in the determination of the function $g$, we used two different forms of it, following Wang (1996) and Ghosh \& Lamb (1991). In the integration scheme, equation (6) is modified accordingly, to include the magnetic coupling.

\section{The minimum mass load as a function of $P$ for a magnetic NS}

We compute the $P$ vs $M_{a c}$ relation for the case of an unmagnetized NS (as in CSTa) considering three selected EoSs. As illustrated in Fig. 3, the spin period $P$ shows a steep dependence on the mass loading 
just above $\sim 3 \mathrm{~ms}$ and small values of $M_{a c}\left(0.04-0.06 M_{\odot}\right)$ are sufficient to produce a millisecond NS. By contrast, substantial mass load is requested for spinning the NS below the observed minimum period $P_{\text {min }}^{\text {obs }}=1.558 \mathrm{~ms}$ (PSR B1937+21). When approaching the limiting period $P_{\text {lim }}$ for mass shedding, the relation $P$ vs $M_{a c}$ flattens. is $\sim 0.05 M_{\odot}$. As already noted by CSTa, all EoSs (labeled as in Arnett \& Bowers 1977) allow a NS to be spun up to $P_{\text {min }}^{o b s}$, but a stiffer EoS requires a higher value of $M_{a c}: 0.25 M_{\odot}$ for $\operatorname{EoS~L~(stiff)~as~compared~to~} 0.1 M_{\odot}$ for $\operatorname{EoS} \mathrm{A}$ (soft). We have verified that such figures are only slightly affected by assuming a lower initial gravitational mass for the NS (e.g. $\left.1.30 M_{\odot}-1.35 M_{\odot}\right)$.

In the case of a magnetized NS, the rate at which angular momentum is transferred to the NS is controlled by the interaction of the magnetosphere with the inner boundary of the accretion disk. Thus, the mass load $M_{a c}$ which is necessary to attain a period $P$ depends upon the magnitude of the $B$-field, on its evolution and on the torque function $\mathrm{g}(\Omega)$. We describe different evolutionary pathways, during recycling, introducing two characteristic times: the spinup time $\tau_{u p}=P / \dot{P}$ and $\tau_{\mu}=\mu / \dot{\mu}$, the timescale of decay of the star's magnetic moment $\mu$.

As a guideline we consider (i) a two-steps evolution in which a phase of significant $\mu$-decay (with $\tau_{\mu} \ll \tau_{u p}$ ) precedes the phase of spinup at constant $\mu$ (with $\tau_{\mu} \gg \tau_{u p}$ ); and (ii) evolutionary pathways with $\tau_{\mu}=(6 / 7) \tau_{u p}$. This condition describes the idealized situation of a NS sliding parallel to the spinup line (corresponding to a fixed accretion rate) without braking along it. In other words, the decay of $\mu$ is tuned just to allow for the NS to approach but never reach the equilibrium period, where the efficiency of the spinup process drops steeply (Wang 1996, Ghosh \& Lamb 1991). In fact, at the equilibrium period, the star would accrete matter without increasing its rotational rate. It is easy to demonstrate that the mass necessary to attain a final period $P$ in case (i) exceeds that of case (ii) by a factor $4 / 3$. For $\tau_{\mu} \gg \tau_{u p}$ even larger amounts of mass are needed. Thus, case (ii) provides a lower limit on the mass load onto the NS, during the recycling process.

The relativistic corrections at short rotational periods and the details of the disk-magnetosphere interaction prevent a simple analytical study of the spin evolution. Hence, we explored numerically the NS evolution. Fig. 4 collects a sample of pathways in the plane $P$ vs $M_{a c}$ obtained for the equation of state FPS (an intermediate EoS), combining various initial conditions (periods in the range $1-100$ sec, $\mu_{i n i}$ between $10^{28}-10^{30} \mathrm{G} \mathrm{cm}^{3}$, accretion rates in the interval $0.01-1.00 \dot{M}_{E d d}$ ) and selecting decay histories according to models (i) and (ii). As illustrated in Fig. 4, the values requested to drive a NS to a final period $P$ depend on the magnetic field evolution. We notice however, that only for NSs with $P>5$ ms at the end of binary evolution the mass load is sensitive to the history of the $\mu$-field. For a magnetized NS the demand of accreted matter can at most halve, relative to the value inferred for a unmagnetized NS. If evolution proceeds to drive $P$ below $5 \mathrm{~ms}$, the request of $M_{a c}$ depends weakly on the evolution of $\mu$ since the magnetospheric radius shrinks, becoming comparable to the radius of the last marginally stable orbit or to the physical radius of the star. Therefore, the $P$ vs $M_{a c}$ relation relaxes to the line that characterizes a unmagnetized NS.

\section{Discussion}

Millisecond pulsars (MSPs) with $P<1 \mathrm{~ms}$ have not been detected so far. Though such sources are beyond the sensitivity limits of the radio surveys conducted until now, available data may already place

constraints on the distribution of MSPs at periods shorter than $P_{m i n}^{o b s}$. Through consideration of surveys sensitivities and known selection effects, Cordes \& Chernoff (1997) carried out a likelyhood analysis and 
found that the best-fit models are those increasing towards short periods with a best-fit minimum period that lies only slightly below $P_{m i n}^{o b s}$. They in addition found a $95 \%$ chance that the fastest MSPs are slower than $1 \mathrm{~ms}$, and a 1\% chance that they are as fast as $0.65 \mathrm{~ms}$. Recently, Possenti et al. (1998) carried out a population synthesis calculation to determine the fraction of neutron stars with periods shorter than $P_{\min }^{\text {obs }}$ relative to those having $P>P_{\text {min }}^{o b s}$, for $\mu>7.3 \times 10^{25} \mathrm{Gcm}^{3}$ (the minimum magnetic moment observed so far). They found that the process of recycling in low mass binaries can lead to a distribution of periods

extending below $P_{\min }^{o b s}$ (for the soft EoS a maximum is found to develop about $1 \mathrm{~ms}$; Possenti et al. 1999, in preparation). If future observations (as those undergoing at the Northern Cross in Medicina: D'Amico et al. 1998) will reveal such pulsars, the underlying NSs should have accreted at least $0.3 M_{\odot}$.

Few estimates of the masses of MSPs in low mass binaries are now available and are reported in Fig. 5. The data, taken from Thorsett \& Chakrabarty (1998), are all compatible with the $P$ vs $M_{a c}$ relation derived in $\S 3$. In particular for PSR B1855+09, the MSP with a mass estimate based on GR alone $\left(M_{G}=1.41 \pm 0.1 M_{\odot}\right)$, the (minimum) mass requested to reach the observed period $(P=5.362 \mathrm{~ms})$ would be of at most $0.06 M_{\odot}$ (for the stiff EoS and an initially low value of $\mu$ ).

Thorsett \& Chakrabarty (1998) found that data obtained collecting the mass measurements of all the known radio pulsars in binary systems are consistent with a remarkably narrow underlying gaussian mass distribution, with $M_{G}=1.35 \pm 0.04 M_{\odot}$. Their sample of objects contains however five relativistic NS-NS binary systems that contaminates the statistics of MSP binaries, narrowing the value of $M$ and in turn of $M_{a c}$. The progenitors of the NS-NS systems are massive X-ray binaries, a population which does not lead to the formation of fast spinning pulsars. If the proposed value of $M_{a c}<0.1 M_{\odot}$ is representative of the MSPs observed sample, this figure is suggestive that a fast decay of the magnetic field has occurred in these systems (not only in PSR B1855+09). This is not in contraddiction with our estimates on $M_{a c}$ because only for those MSPs recycled to limiting periods $P<P_{m i n}^{o b s}$ the demand of mass load would exceed that of Thorsett \& Chakrabarty.

Interestingly, a suggestion of the existence of NSs with masses larger than $1.4 M_{\odot}$ arises also from a recent model (Stella \& Vietri 1999) accounting for the pair kHz-QPOs observed in many LMXB sources (see e.g. van der Klis 1998 for a review). Stella \& Vietri interprete the upper QPO frequency $\nu_{2}$ as due to matter inhomogeneities orbiting the NS at the inner disk boundary, while the lower QPO frequency $\nu_{1}$ is produced by the periastron precession at the inner edge of the accretion disk. In this framework, the observed clustering of the differences $\Delta \nu=\nu_{2}-\nu_{1}$ around $250-360 \mathrm{~Hz}$ is naturally explained provided the gravitational mass of the typical accreting NSs is $\sim 2 M_{\odot}$. Moreover the detection of millisecon X-ray pulsations from SAX J1808.4-3658 at different luminosity levels of the source suggests the presence of a massive NS in this system as discussed by Burderi and King (1998).

\section{Conclusion}

So far, there is no observational evidence against the existence of submillisecond NSs. In the evolutionary scheme adopted, the formation of submillisecond NSs is a natural outcome if a minimum mass of $\simeq 0.25-0.30 M_{\odot}$ is accreted and if the magnetic moment decays down to values $10^{25}-10^{26} \mathrm{Gcm}^{3}$ before the end of recycling.

Only highly non-conservative scenarios may indeed prevent the transfer of $M_{a c} \geq 0.3 M_{\odot}$ in low-mass binaries. In fact, evolutionary considerations shows that the mass transfer occurs either because of the nuclear evolution or because angular momentum losses of a $\sim 1 M_{\odot}$ companion (see e.g. Burderi, King 
\& Wynn 1996; Webbink, Rappaport \& Savonije 1983). A more massive companion $\left(M \gtrsim 2 M_{\odot}\right.$ - if we retain the hypothesis that the NS mass at birth is $\sim 1.4 M_{\odot}$ ) would determine a dynamically unstable mass transfer probably ending into a common envelope phase (see Kalogera \& Webbink 1996; 1998). On the other hand, a low mass companion $\left(M \lesssim 1 M_{\odot}\right)$ would either underfill its Roche lobe, because its nuclear evolution timescale is longer than the age of the Galaxy for initial orbital periods $\gtrsim 0.5$ days, or, for shorter initial orbital periods, would fills its Roche lobe during its main sequence lifetime, resulting in a near-permanent faint LMXB whose compact object will never appear as a radiopulsar. Therefore, in order to produce a fastly rotating NS, we are restricted to a quite narrow range of companion masses, (namely $1-2 M_{\odot}$ ), and of initial orbital periods (namely $0.5-10$ days). For such binary systems, typical timescales for intense accretion are $\lesssim 10^{8}$ years. Once the mass-transfer ceases, the endpoint is often a circular binary containing a millisecond radio pulsar and a low-mass $\left(\sim 0.2-0.3 M_{\odot}\right)$ white dwarf companion, the latter being the degenerate helium core of the sub-giant which transferred mass to the compact object (see e.g. Burderi, King \& Wynn 1998). This implies that the companion has lost $\gtrsim 1 M_{\odot}$ during the evolution of the system. Even taking into account the case of an highly non-conservative mass transfer phase, it is difficult to believe that the NS in these systems has accreted less than $0.3 M_{\odot}$.

There might be a number of physical processes that inhibit the formation of sub-MSPs during binary evolution.

(a) Slow decay of the magnetic moment would relent the spinup process thus requiring a amount of mass exceedingly large. In addition, precocious freezing of $\mu$ (above $10^{27} \mathrm{G} \mathrm{cm}^{3}$; as discussed in Konar \& Bhattacharya 1997; 1998) may prevent the formation of very fastly spinning NSs.

(b) Long term variation of the accretion rate may be important. The terminal phases of LMXB evolution are still uncertain depending on the Roche-Lobe feeding mechanisms. If the accretion rate ceases on a time comparable to the evolutionary time scale, the propeller effect may cause an increase of the spin period above $P_{m i n}^{o b s}$. Approximately, the ratio of the spin down time $\tau_{s d}$ by propeller to $\tau_{u p}$ scales as

$$
\frac{\tau_{s d}}{\tau_{u p}}=\left(\frac{\dot{m}_{u p}}{\dot{m}_{s d}}\right)^{5 / 7}\left(\frac{\mu_{u p}}{\mu_{s d}}\right)^{2 / 7} \frac{1}{\xi}
$$

where indexes up and $s d$ refer to spinup and spindown, and $\xi$ is a parameter measuring the efficiency of the extraction of angular momentum from the NS during the propeller phase $(\xi=1.0$ if the efficiency of spin up and down are equal). For spindown to be negligible, that ratio needs to exceed unity, requiring a rather rapid turn off of the mass transfer rate $\left(\dot{m}_{s d} \ll \dot{m}_{u p}\right)$ (Possenti et al. in preparation).

(c) Similar considerations holds in case in which the binary is subject to disk instabilities (Cannizzo 1993 and references therein), leading to long periods of low level of activity - referred in literature as quiescence - interrupted by brief periods of outbursts (van Paradijs 1996, King 1998). For the known soft X-ray transients (SXTs: see Campana et al. 1998 for a complete review) the first factor in equation (13) is $\sim 100$, whilst the ratio of $\mu$ 's stays nearly constant during a cycle. If the quiescence phase lasts $\sim 100 / \xi$ times longer than the outburst phase, spin down would prevail. The off-on time ratio of observed recurrent transients varies from source to source and is about $10-100$. It is thus difficult to assess the relevance of this process in affecting the spin evolution and the statistical properties of the MSP population. Recently Li, van den Heuvel \& Wang (1998) argued that unsteady mass transfer in PSR J1455-3330 may have occurred, and explored evolutionary tracks allowing for spindown by propeller during the long quiescent intervals that the binary would experience if fed through a thermally unstable keplerian disk. The discovery of SAX J1808-3658 might challenge this picture.

(d) For NSs with hot interiors, the viscosity of the nuclear matter may become inefficient in damping the 
recently discovered $r$-mode instabilities of the star (Andersson 1998). The related emission of gravitational waves (Stergioulas 1998) could limit the spin of a NS to a small fraction $(\sim 0.2-0.3)$ of its mass shedding rotational rate. For young NSs with $T>10^{9}$, this effect could spindown the star at $P>10 \mathrm{~ms}$ in a very short time ( $\sim 1$ yr) (see e.g. Lindblom, Owen \& Morsink 1998). Andersson, Kokkotas \& Stergioulas (1998) claimed that a similar mechanism could occur during the LMXB-phase, thus preventing the spinup of a NS below $\sim P_{\text {min }}^{o b s}$. However, the difficulties in the calculations of the values of the shear and bulk viscosity for superfluid nuclear matter at $T \sim 10^{7}-10^{8.5} \mathrm{~K}$ (relevant for the accretion phase) leave a large uncertainty in the role of this instability in the recycling process. In addition, crustal deformations induced by accretion can excite emission of gravitational waves inhibiting the spin-up process to period shorter than $\sim 3 \mathrm{~ms}$ (Bildsten 1998).

\section{REFERENCES}

Andersson, N. 1998, ApJ, in press, gr-qc/9706075

Andersson, N., Kokkotas, K.D., Stergioulas, N. 1998, astro-ph/9806089

Arnett, W.D., Bowers, R.L. 1977, ApJS, 33, 415

Bardeen, J.M., Press, W. H., Teukolsky, S. A. 1972, ApJ, 178, 347

Bhattacharya, D., van den Heuvel, E. P. J. 1991, Phys. Rep., 203, 1\&2, 1

Bildsten, L. 1998, ApJ, 501, L89

Burderi, L., Di Salvo, T., Robba, N. R., Del Sordo, S., Santangelo, A., Segreto, A. 1998, ApJ, 498,831

Burderi, L., King, A. R., Wynn, G. A. 1996, ApJ, 457, 348

Burderi, L., King, A. R., Wynn, G. A. 1998, MNRAS, in press

Burderi, L., King, A. R. 1998, ApJ, 505, L135

Campana, S., Colpi, M., Mereghetti, S., Stella, L., Tavani, M. 1998, Ann.Rev. A\&A, in press

Cannizzo, J.K. 1993, Accretion Disks in Compact Stellar Systems, ed. J.C. Wheeler, (Singapore: World Scientific Publishing), 6

Cook, G.B., Shapiro, S.L., Teukolsky, S.A. 1994a, ApJ, 423, L117 (CSTa)

Cook, G.B., Shapiro, S.L., Teukolsky, S.A. 1994b, ApJ, 424, 823 (CSTb)

Cordes, J.M., \& Chernoff, D.F. 1997, ApJ, 482, 971

D'Amico N., et al. 1998, Proc. Neutron Stars and Pulsars Symp., eds. Shibazaki, N., et al. (Tokyo), 235

Ghosh, P., \& Lamb, F.K. 1991, in Proc. NATO/ASI Ser 344, Neutron Stars: Theory and Observations, ed. J.Ventura \& D.Pines (Dordrecht: Kluwer), 363

Kalogera, V., \& Webbink, R.F. 1996, ApJ, 458, 301

Kalogera, V., \& Webbink, R.F. 1998, ApJ, 493, 351 
King, A.R. 1998, MNRAS, 296, L45

Konar, S., \& Bhattacharya, D. 1997, MNRAS, 284, 311

Konar, S., \& Bhattacharya, D. 1998, MNRAS in press, astro-ph/9808119

Li, X.D., van den Heuvel, E.P.J., Wang, Z.R. 1998, ApJ, 497, 865

Lindblom, L., Owen, B.J., Morsink, S.M. 1998, to appear in Phys.Rev.Lett., gr-qc/9803053

Lipunov, V.M. 1992, Astrophysics of neutron stars (Springer Verlag)

Mendez, M., van der Klis, R., Wijnands, R., Ford, E.C., van Paradijs, J., Vaughan, B.A. 1998, submitted, astro-ph/9807281

Phinney, E.S., Kulkarni, S.R. 1994, Ann.Rev.Astron.Astrophys., 32, 591

Portegies Zwart, S.F., Yungelson, L.R. 1998, A\&A, 332, 173

Possenti, A., Colpi, M., D’Amico, N., Burderi, L. 1998, ApJ, 497, L97

Ravenhall, D.G., Pethick, C.J. 1994, ApJ, 424, 846

Shapiro S.L., Teukolsky, S.A. 1983, Black Holes, White Dwarfs and Neutron Stars (J.Wiley \& Sons: New York), 178

Shibata, M., Sasaki, M. 1998, submitted, gr-qc/9807046

Stella, L. \& Vietri, M. 1998, astro-ph/9812124, to appear in Phys. Rev. Let.

Stergioulas, N. 1998, Rotating Stars in Relativity, Living Reviews in Relativity, gr-qc/9805012

Thorsett, S.E., Chakrabarty, D. 1998, ApJ submitted, astro-ph/9803260

Timmes, F.X., Woosley, S.E., Weaver, T.A. 1996, ApJ, 457, 834

van der Klis, M. 1998, The many faces of neutron stars,eds. R.Buccheri, J. van Paradijs, M.A. Alpar (Dordrecht: Kluwer Academis Publisher), in press

van den Heuvel, E.P.S., Rappaport, S.A. 1992, X-ray Binaries and the Formation of Binary and Millisecond Pulsars, (Dordrecht: Kluwer)

van Paradijs, J. 1996, ApJ, 464, L139

Webbink, R.F., Rappaport, S., Savonije, G.J. 1983, ApJ, 270, 678

Werbunt, F. 1993, Ann.Rev.Astron.Astrophys., 31, 93

Woosley, S.E., Weaver, T.A. 1986, Ann.Rev.Astron.Astrophys., 24, 205

Wang, Y. 1996, ApJ, 465, L111 


\begin{tabular}{c|c|c|c}
\hline \hline EoS & $\begin{array}{c}P_{\lim }(\mathrm{ms}) \\
\text { (this work) }\end{array}$ & $\begin{array}{c}P_{\lim }(\mathrm{ms}) \\
\text { (Cook et al., 1994a) }\end{array}$ & $\begin{array}{c}\text { Difference } \\
(\%)\end{array}$ \\
\hline $\mathrm{A}$ & 0.631 & 0.604 & 4.5 \\
$\mathrm{C}$ & 0.907 & 0.894 & 1.4 \\
$\mathrm{D}$ & 0.751 & 0.730 & 2.8 \\
$\mathrm{E}$ & 0.677 & 0.656 & 3.1 \\
$\mathrm{~F}$ & 0.679 & 0.715 & -5.1 \\
$\mathrm{~L}$ & 1.21 & 1.25 & -2.9 \\
$\mathrm{M}$ & 1.48 & 1.49 & -0.9 \\
$\mathrm{~N}^{*}$ & 1.03 & 1.08 & -4.7 \\
$\mathrm{KC}$ & 0.910 & 0.888 & 2.5 \\
$\mathrm{AU}$ & 0.708 & 0.701 & 1.0 \\
$\mathrm{UU}$ & 0.792 & 0.784 & 1.0 \\
$\mathrm{UT}$ & 0.764 & 0.754 & 1.4 \\
$\mathrm{FPS}$ & 0.767 & 0.747 & 2.8 \\
\hline \hline
\end{tabular}

Table 1: Minimum rotational periods for the EoSs indicated in column 1. In column 2 we give the periods obtained from the analytical model derived in this work. In column 3 we collect the numerical values from Cook, Shapiro, \& Teukolsky (1994a). The fourth column indicates the percentage differences. 


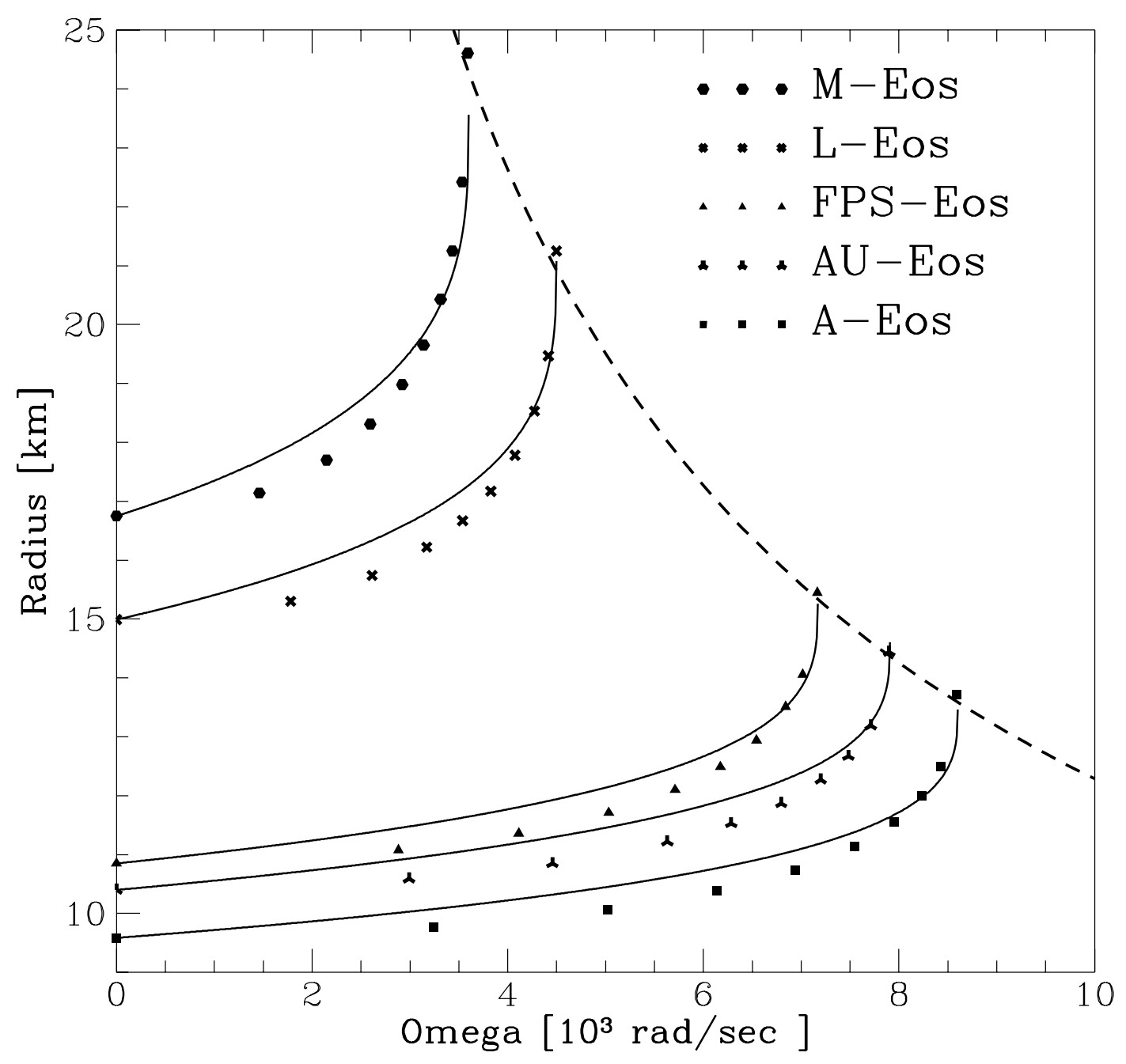

Fig. 1. - NS circumferential radii (in units of km) versus angular frequency $\Omega$ (in units of $10^{3} \mathrm{rad} / \mathrm{sec}$ ). The points are from Cook, Shapiro \& Teukolsky (1994b) for 5 representative EoSs $\left(M_{B}=1.4 M_{\odot}\right)$. The solid lines are relation (5). The dashed line is the keplerian relation (3). 


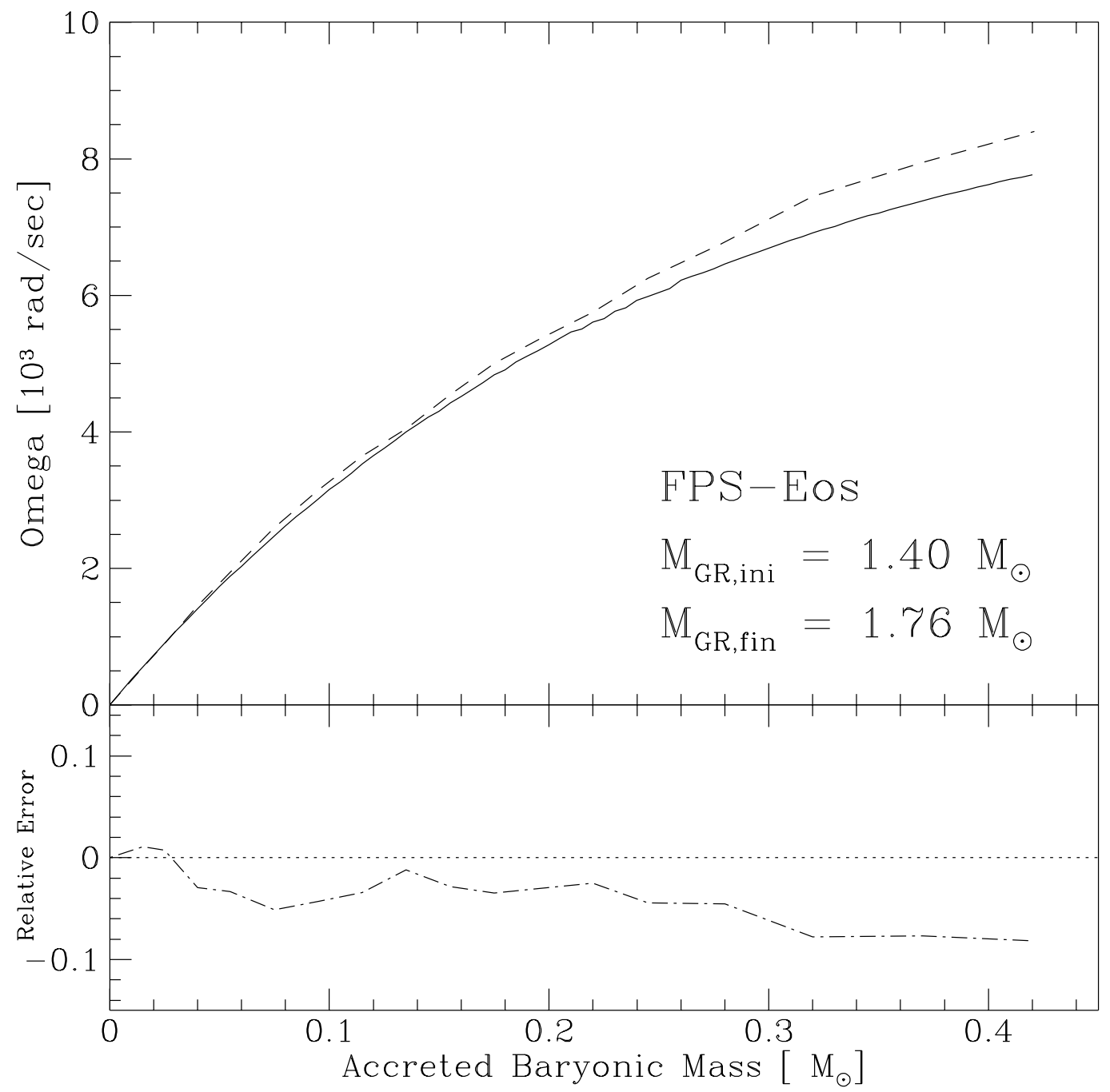

Fig. 2.- Rotational evolution of an unmagnetized NS (initial gravitational mass $M_{G, i n i}=1.40 M_{\odot}$ ) undergoing steady accretion of matter. The $\Omega$ vs $M_{a c}$ relation calculated for FPS-EoS with the semianalytic model described in the text (solid line) is compared to that from CSTa (dashed line). The relative errors in computing $\Omega$ with our approximate model are displayed in the lower panel. Accretion is halted at $M_{a c}=0.416 M_{\odot}$, when mass shedding sets in (the final gravitational mass of the NS is $M_{G, f i n}=1.76 M_{\odot}$ ). 


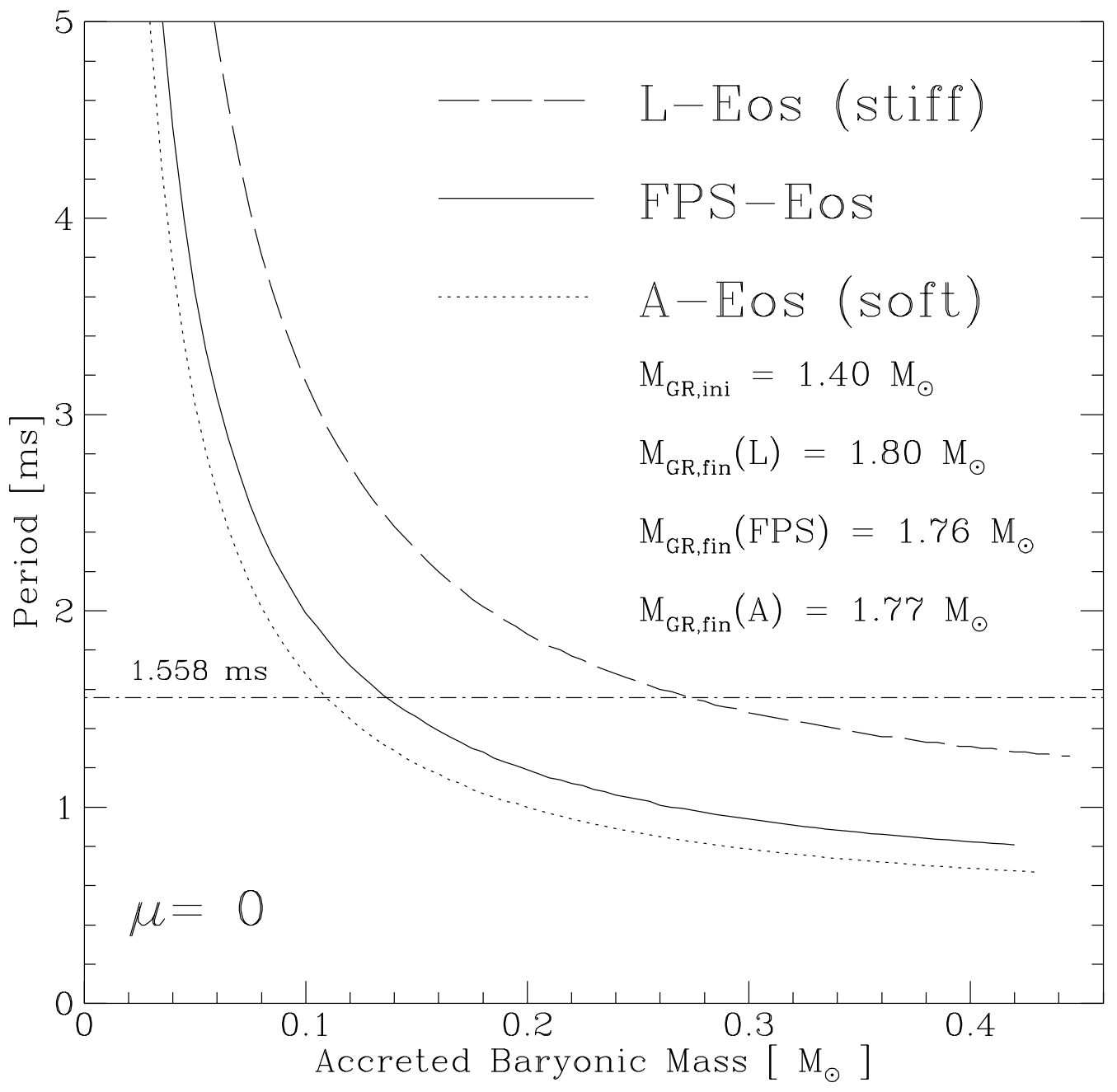

Fig. 3.- $P$ vs $M_{a c}$ relations for the case of a unmagnetized NS considering three selected equations of state: L (stiff: dashed line), A (soft: dotted line), FPS (intermediate: solid line). The initial gravitational mass of the NS is always $1.40 M_{\odot}$. The period $P$ is measured in millisecond, the accreted baryonic mass $M_{a c}$ is in solar mass. The dashed-dotted line represents the minimum rotational period observed so far. Accretion is halted when the centrifugal limit is reached. 


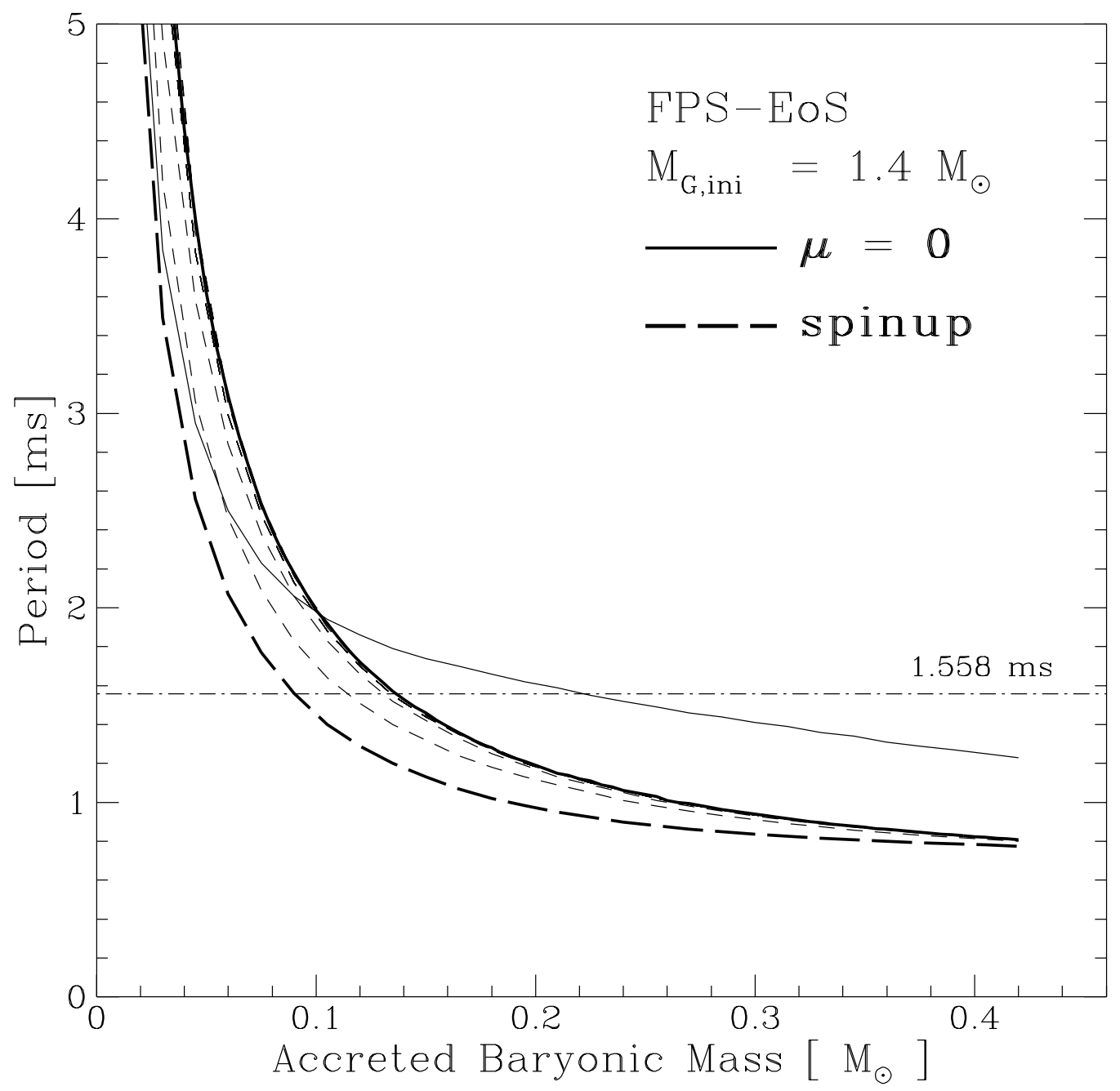

Fig. 4.- $P$ vs $M_{a c}$ relations for a magnetized NS using FPS EoS and an initial gravitational mass of $1.40 M_{\odot}$. The different pathways (a sample is represented by the dashed lines) define a strip, which narrows towards shorter periods (see text for details). The strip is upper bounded by the evolutionary path for an unmagnetized NS (bold solid line). The bold long dashed line refers to the evolution along the spinup line and is calculated assuming a step shaped $g$-torque function (an extreme situation which maximizes the efficiency of the spinup process). The thin solid line represents the effect of a slow $\mu$-decay: the evolution along the spinup line is "braked" and the requested mass load increases. 


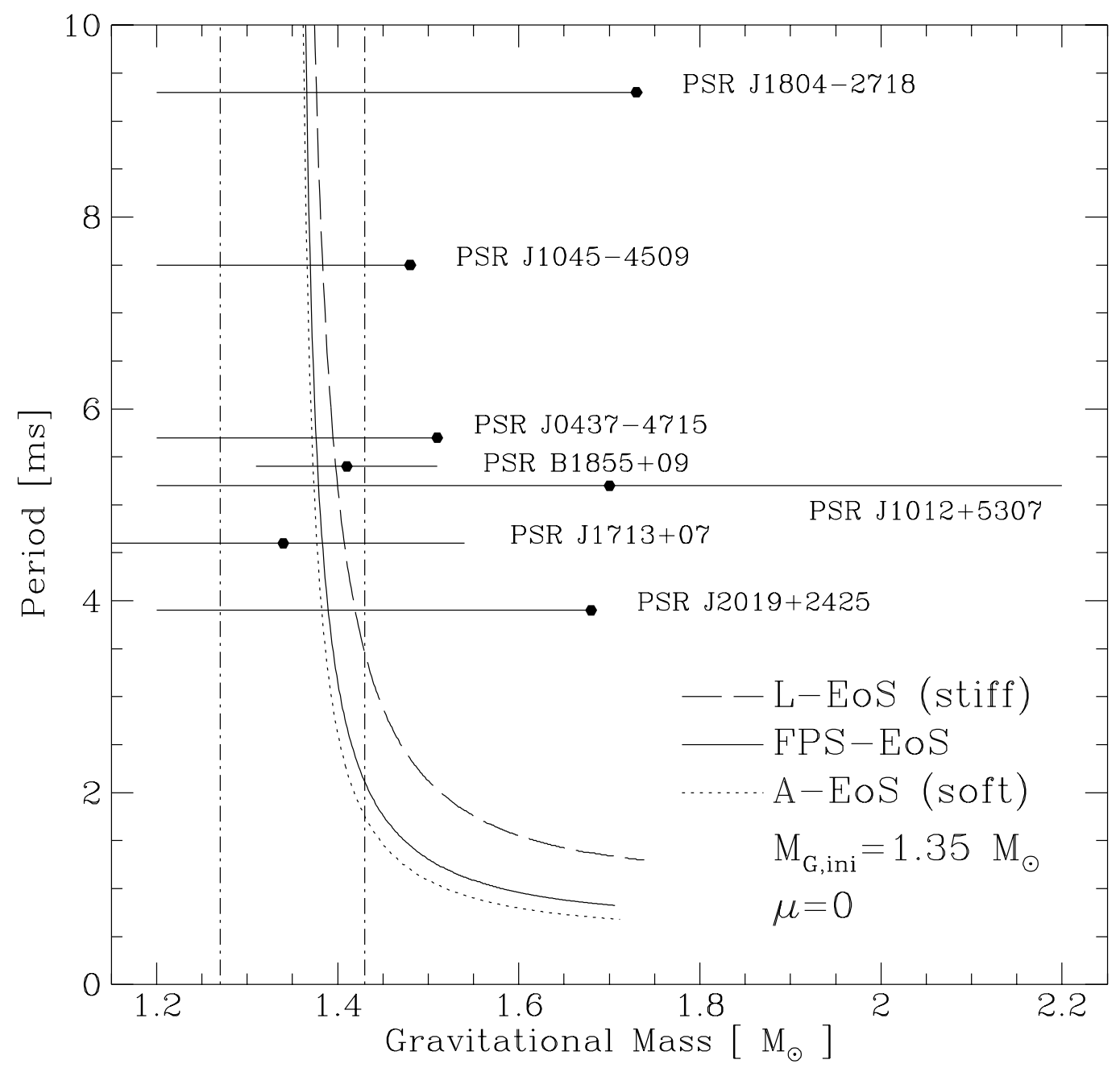

Fig. 5.- Available mass estimates for MSPs $(P<10 \mathrm{~ms})$ compared with the evolutionary lines labeled as in Fig. 3, but calculated for an initial gravitational mass of $M_{G, i n i}=1.35 M_{\odot}$. The error bars indicate central $68 \%$ confidence limits, while upper limits are one-sided $95 \%$ confidence limits. All the estimates are from Thorsett \& Chakrabarty (1998). Vertical lines bind the $2 \sigma$-confidence region for the NSs masses $\left(M_{G}=1.35 \pm 0.08\right)$ derived by the same authors. 